University of Minnesota, Minneapolis, Minn.

University of Missouri, Columbia, Mo.

University of Nebraska, Lincoln, Neb.

University of New Mexico, Albuquerque, N.Mex.

New York University, New York, N.Y.

University of North Carolina, Chapel Hill, N.C.

Northwestern University, Evanston, Ill.

University of Notre Dame, Notre Dame, Ind.

University of Oklahoma, Norman, Okla.

Oregon General Research Council, Corvallis and Eugene, Ore.

Pennsylvania State College, State College, $\mathrm{Pa}$.

University of Pennsylvania, Philadelphia, $\mathrm{Pa}$.

University of Pittsburgh, Pittsburgh, $\mathrm{Pa}$.

Purdue University, Lafayette, Ind.

University of Rochester, Rochester, N.Y.

Rutgers University, New Brunswick, N.J.

The College of St. Thomas, St. Paul, Minn.

Smith College, Northampton, Mass.

University of Southern California, Los Angeles, Calif.

Stanford University, Stanford University, Calif.

Stevens Institute of Technology, Hoboken, N.J.

Swarthmore College, Swarthmore, $\mathrm{Pa}$.

Sweet Briar College, Sweet Briar, Va.

Syracuse University, Syracuse, N.Y.

University of Toronto, Toronto, Ontario, Canada.

Trinity College, Hartford, Conn.

Tulane University, New Orleans, La.

Vassar College, Poughkeepsie, N.Y.

Virginia Polytechnic Institute, Blacksburg, Va.

University of Virginia, Charlottesville, Va.

University of Washington, Seattle, Wash.

Washington University, St. Louis, Mo.

Wayne University, Detroit, Mich.

Wellesley College, Wellesley, Mass.

Wells College, Aurora, N.Y.

Wesleyan University, Middletown, Conn.

Western and Southern Life Insurance Company, Cincinnati, Ohio.

Western Reserve University, Cleveland, Ohio.

Williams College, Williamstown, Mass.

Yale University, New Haven, Conn.

\title{
ORGANIZATIONS COÖPERATING AS NON MEMBERS
}

Lehigh University, Bethlehem, Pa.

National Research Council, Washington, D.C.

Princeton University, Princeton, N.J.

Rockefeller Foundation, New York, N.Y.

University of Wisconsin, Madison, Wis. 